\title{
Die Notwendigkeit der ökologischen Analyse und Bewertung wirtschaftlicher Aktivitäten
}

\author{
Mario Schmidt
}

Online veröffentlicht: 20.5.2009

(C) Springer-Verlag 2009

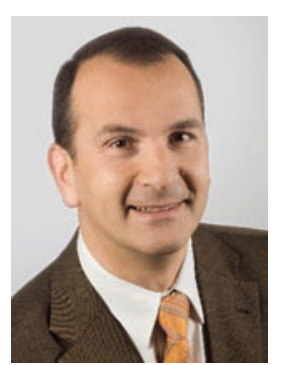

In der Fachwelt werden derzeit intensiv die Entwicklung und der Einsatz von so genannten Carbon Footprints diskutiert. Damit soll ermöglicht werden, die Treibhauswirkung von Produkten über den ganzen Lebensweg hinweg zu beziffern. Die Internationale Standardisierungsorganisation (ISO) hat zur Erstellung einer Carbon-Footprint-Norm sogar einen entsprechenden Ausschuss eingerichtet. Doch diese Planungen sind sehr umstritten. Auf der einen Seite wird der Carbon Footprint als inhaltlich wenig aussagekräftig gegenüber dem methodisch wesentlich ausgereifteren Konzept der Ökobilanz/Life Cycle Assessment (LCA) angesehen. LCA-Protagonisten reden gar von einer „LCA für Arme". Man befürchtet einen Missbrauch des Carbon Footprints im Bereich der Produktkennzeichnung. Auf der anderen Seite hat der Carbon Footprint für die Anwender in der Wirtschaft einigen Charme. Er lässt sich mit vertretbarem Aufwand durchführen und fokussiert auf jenes Umweltthema, das politisch die höchste Priorität derzeit hat: auf den

Prof. Dr. Mario Schmidt $(\bowtie)$

Institut für Angewandte Forschung/Fakultät für Wirtschaft und Recht Hochschule Pforzheim

Tiefenbronner Str. 65

Pforzheim, Deutschland

E-Mail: mario.schmidt@hs-pforzheim.de
Klimaschutz. So wundert es nicht, dass Hersteller, Handel und Berater im Carbon Footprint ein großes Potenzial am Markt sehen - was selten genug für ein Umweltthema vorkommt.

Das Beispiel zeigt eines sehr deutlich: Es werden weiterhin Methoden für die Analyse und Bewertung der Umweltwirkungen von unternehmerischen Aktivitäten benötigt. Dieser Bedarf besteht insbesondere für eine breite (!) Anwendung in der Wirtschaft, unter Einbeziehung der komplex strukturierten und international ausgerichteten Supply Chains. Und sie müssen sich in die Entscheidungsabläufe von Unternehmen integrieren lassen. Die LCA kann immerhin auf mehrere Jahrzehnte an Erfahrung, Methodenentwicklung und Normierung zurückblicken. Aber sie hat auch den offensichtlichen Nachteil, dass ihre Erstellung ausgesprochenen Experten vorbehalten bleibt, die Datenakquisition sehr aufwendig ist und die Interpretation der differenzierten Ergebnisse nicht trivial ist. Sie ist weit von der betriebswirtschaftlichen Praxis entfernt. Sie wird auch in Zukunft kaum auf breiter Front eingesetzt, sondern eher auf besonders wichtige Produktbereiche fokussiert werden.

An diesem Punkt setzt dieses uwf-Schwerpunktheft an. Es handelt von einer Methode, bei der eine Umweltleistungszahl für betriebliche Aktivitäten am Beispiel der Treibhauswirkung entwickelt wird, die anhand realer und regelmäßig aktualisierter Daten mit vertretbarem Aufwand erzeugt werden kann. Sie kann auf Unternehmen und auf Produkte angewendet werden. Entscheidend ist dabei nicht, dass es sich um nur eine Zahl handelt - das Konzept ließe sich auch auf weitere ökologische Wirkungskategorien erweitern. Aber die „,kumulierte Emissionsintensität“ (KEI) erfüllt den Anspruch, die „Vorketten“ oder „ökologischen Rucksäcke“ mit zu berücksichtigen, und baut zusätzlich auf dem Prinzip auf, dass ein Unternehmen höchstens die Daten seiner unmittelbaren Lieferanten beschaffen kann. Dies 
entspricht der betrieblichen Praxis, denn welches Unternehmen kann schon - wie bei der LCA - jedes Mal zurück bis zur Ressourcenentnahme aus der Umwelt bzw. vorwärts bis zur Deponierung des Produktes Primärdaten zu seinem Produktlebensweg sammeln. Die Alternative dazu sind generische Daten mit begrenztem Wert für unternehmerische Entscheidungen.

Unternehmen sind mit konkreten Lieferbeziehungen und Produktionsbedingungen konfrontiert; diese müssen sie analysieren und optimieren. Auch der vorgestellte Ansatz bewegt sich im Spannungsfeld zwischen der Datenakquisition mit einem vertretbaren Aufwand und der Ableitung trotzdem belastbarer Ergebnisse. Dies führt zu Unterschieden gegenüber den gängigen Verfahren wie der LCA, zu Vor- und Nachteilen. Wir wollen nicht behaupten, dass die kumulierte Emissionsintensität der Weisheit letzter Schluss ist. Aber die Auseinandersetzung mit diesem Thema, mit den Anforderungen, mit den sinnvollen und den weniger zweckmäßigen Annahmen halten wir für geboten, wenn es gelingen soll, dass solche Verfahren in der Wirtschaft stärker eingesetzt werden und nicht einer kleinen Expertengemeinde vorbehalten bleiben.

Man kann darüber diskutieren, ob es langfristig überhaupt notwendig sein wird, die Umweltwirkungen wirtschaftlicher Tätigkeiten explizit und ausführlich zu quantifizieren, oder ob es nicht ausreicht, die Umweltwirkungen durch entsprechende Emissionsabgaben oder Steuern zu internalisieren und nur über den Preis der Produkte abzubilden. Aus betrieblicher Sicht wird es aber immer interessant sein, die Zusammensetzung von Preisen oder Umweltbelastungen im Einzelnen zu analysieren, in Entscheidungsprozesse einfließen zu lassen und die Systeme dann entsprechend zu optimieren. Zum Beispiel ist die Berücksichtigung der Umweltleistung von Lieferanten und der damit verbundenen ökonomischen Risiken ein wichtiger Aspekt, für den geeignete Verfahren noch ausstehen. Selbst wenn z. B. die Treibhausgasemissionen bei der Preisbildung der Lieferanten berücksichtigt wären, bliebe unklar, wie volatil die Preise mit steigenden $\mathrm{CO}_{2}$ - oder Energiekostenanteilen sind. Zusätzliche Kenngrößen auf der Basis von Mengenangaben wären hier hilfreich.

Die Idee mit den kumulierten Emissionsintensitäten stammt ursprünglich aus einer ganz praktischen Fragestellung in der Wirtschaft. Ein Automobilkonzern wollte die Treibhauswirkung verschiedener Produktionsstandorte miteinander vergleichen. Doch wie berücksichtigt man die verschiedenen Produktprogramme, die unterschiedlichen Fertigungstiefen und die spezifische Lieferantenstruktur der einzelnen Standorte? Wie kann man Ungleiches vergleichbar machen? An der Hochschule Pforzheim entstand die Grundidee, die Wertschöpfung als Bezugspunkt zu nutzen, was schließlich auf den vorgestellten Ansatz führte. Das baden-württembergische Wissenschaftsministerium förder- te die Arbeiten zunächst als so genanntes „innovatives Projekt“. Im Jahr 2006 wurde mit dem Verbundvorhaben EINBLIK - Kumulierte Emissionsintensitäten zur Bewertung der Klimaschutz-Performance entlang von Lieferketten eine Kooperation zwischen der Hochschule Pforzheim, der Technischen Universität Braunschweig, der Volkswagen AG und der Systain GmbH aus der Otto-Gruppe gestartet, um die Methode weiterzuentwickeln und in der Praxis zu testen. EINBLIK ist damit eines von ca. 40 anwendungsorientierten Forschungsprojekten, die vom Bundesministerium für Bildung und Forschung (BMBF) im Rahmen des Förderschwerpunktes „klimazwei - Forschung für den Klimaschutz und Schutz vor Klimawirkungen“" gefördert wurden.

Die Kennzahl der kumulierten Emissionsintensität ist nur beispielhaft auf die Treibhauswirkung bezogen; sie könnte auch auf andere ökologische Wirkungsbereiche ausgedehnt werden. Außerdem ist sie nicht an die Anwendung in einer speziellen Branche gebunden. Die von den Praxispartnern vertretenen Branchen der Textil- und Automobilindustrie stellen für das Projekt lediglich idealtypische Wertschöpfungsketten dar, denn die betrachteten Praxisfälle bilden eine Vielzahl von Komplexitätsgraden ab. So zeichnet sich die Textilindustrie beispielsweise durch ihre internationale Ausrichtung, die klare Abgrenzungsfähigkeit der einzelnen Wertschöpfungsstufen und ihre fluktuativen Geschäftsbeziehungen aus, während die Automobilindustrie durch komplexe Produkte, eine Vielzahl von Unternehmensstandorten und eine starke Einbindung der Lieferanten gekennzeichnet ist.

Die spannende inhaltliche Diskussion auf Tagungen und vor allem auf dem Ergebnis-Workshop im November 2008 mit teilweise externen Fachleuten führte zu der Idee, das Projekt einem größeren Kreis vorzustellen und damit Kolleginnen und Kollegen zu weiteren Meinungen, Kritik und Ideen anzuregen. Der Herausgeber dieser Zeitschrift, Prof. Dr. Dietfried G. Liesegang, bot dazu dankenswerterweise ein Schwerpunktheft an. Das ermöglicht es, die Diskussion zeitnah durchzuführen, denn internationale Fachzeitschriften haben oft einen erheblichen zeitlichen Verzug bis zur Veröffentlichung. Es wurde versucht, einige konzeptionelle Aspekte vorzustellen, über erste Erfahrungen aus der Praxis zu berichten und externe Kollegen zu Wort kommen zu lassen.

In dem ersten Beitrag dieses Schwerpunktheftes skizziert das Pforzheimer Team die Grundidee der kumulierten Emissionsintensität. Hier werden einige Annahmen vorgestellt und es treten auch schon wichtige Unterschiede zu bisherigen Verfahren auf. Es ergibt sich ein interessanter Ausblick, wenn man etwa die vielzitierten Nachhaltigkeitsdimensionen Umwelt, Ökonomie und Soziales im Blick hat und sich fragt, wie diese miteinander interagieren.

Eine große Hürde des neuen Konzeptes ist, dass es so richtig erst funktioniert, wenn ,alle“ mitmachen, d.h. jeder 
in der Lieferantenkette seine direkten Emissionen ermittelt, mit denen seiner Lieferanten verrechnet und an seine Kunden weiterreicht. Damit entsteht ein Startwertproblem, dem sich Christian Haubach intensiv gewidmet hat. Er zieht Daten aus der umweltökonomischen Gesamtrechnung heran, um branchenbezogene Startwerte zu erzeugen.

Ein ebenfalls wichtiger Punkt sind einheitliche Bilanzierungs- und Abgrenzungsregeln, wenn das System insgesamt funktionieren soll. Wenn dieses Verfahren in der Wirtschaft angewendet werden soll, dann müssen die gebräuchlichen Rechnungslegungsstandards herangezogen werden, was Sabine Walter in ihrem Artikel beleuchtet.

Die Ermittlung von Emissionswerten ist kein Selbstzweck, sondern muss in Relation zur externen Bewertung von Unternehmen und zur innerbetrieblichen Verbesserung gesehen werden. Beide Ebenen sind wichtig, doch bisher nicht miteinander verbunden. D.h. die für die externe Bewertung eingesetzten Indikatoren müssen anschlussfähig zu innerbetrieblichen Entscheidungsprozessen und Optimierungsverfahren sein. Mit der Schließung dieser Lücke befasst sich der Beitrag der Braunschweiger Arbeitsgruppe um Prof. Dr. Thomas Spengler. Es wird auf drei Ebenen ein hierarchisches Konzept zur Entscheidungsunterstützung entwickelt, wobei moderne Methoden der Effizienzmessung für die Bewertung und Verbesserung der kumulierten Emissionsintensität eingesetzt werden. Durch Verknüpfung der Ebenen auf Basis von Trade-Offs gelingt die Abschätzung der Auswirkungen des Einsatzes unternehmensinterner Maßnahmen zur Emissionsminderung auf die kumulierte Emissionsintensität von Standorten und Unternehmen.

Bei den praxisbezogenen Themen wurde eine Unterteilung in die vertikale und horizontale Anwendung der Kennzahl vorgenommen. Mit vertikaler Anwendung ist damit gemeint, dass die Kennzahl entlang der Wertschöpfungskette erzeugt und weitergereicht werden muss. Spannend wird es, wenn solche Ansätze international verfolgt und auch in Ländern eingesetzt werden, deren Wirtschaft anders strukturiert ist. Als Beispiel hierfür bietet sich die textile Kette an, die von der Beratungsfirma Systain aus der Otto-Gruppe in Hamburg untersucht wurde. Die Autoren stellen einige Erfahrungen insbesondere bei der Akquisition und Interpretation von Daten, z. B. in der Türkei, in Griechenland oder Thailand vor.

Mit horizontaler Anwendung ist der Vergleich verschiedener ähnlicher Produktionsstandorte gemeint, bei denen aber trotzdem ein direkter Vergleich durch die differierende Lieferantenstruktur, Fertigungstiefe und Produktpalette erschwert wird. Die Volkswagen AG hat das Verfahren exemplarisch bei einigen ihrer Standorte getestet. Dabei wird auch die Brücke zum Einsatz von Entscheidungshilfen im innerbetrieblichen Verbesserungsprozess, wie sie von den Braunschweiger Kollegen vorgestellt wurden, geschlagen.

Als externe Autoren äußern sich Dr. Moritz Nill von der PricewaterhouseCoopers AG sowie Dr. Ivo Mersiowski von der Dekra Umwelt GmbH zu dem Konzept der kumulierten Emissionsintensitäten. Sie tun es aus verschiedener Perspektive: Dr. Nill sieht den enorm steigenden Bedarf an inhaltlich belastbarer Unternehmensberichterstattung im Treibhausgasbereich und bestätigt die Notwendigkeit, hierzu praktikable Verfahren bereitzustellen. Dr. Mersiowski argumentiert vor dem Hintergrund der LCA-Methodik. Auch er hält Weiterentwicklungen in Richtung Effizienzkenngrößen für notwendig und erinnert zu Recht an die Bedeutung der Nutzungsphase von Produkten.

Wir möchten an dieser Stelle allen Beteiligten für ihr Engagement danken: den externen Autoren, den Kolleginnen und Kollegen im Projekt, besonders aber Christian Haubach, der die Koordinierung dieses Schwerpunktheftes übernommen hat, sowie den Herausgebern dieser Zeitschrift für diese Chance zu einem interessanten Diskurs.

Prof. Dr. Mario Schmidt ist Direktor des Instituts für Angewandte Forschung (IAF) und Professor für ökologische Unternehmensführung und Operations Research an der Fakultät für Wirtschaft der Hochschule Pforzheim. Er leitete das BMBF-geförderte Verbundvorhaben EINBLIK. 